\title{
Uniformly Discrete Forests with Poor Visibility
}

\author{
NOGA $\mathrm{ALON}^{\dagger}$ \\ Sackler School of Mathematics and Blavatnik School of Computer Science, Tel Aviv University, Tel Aviv 69978, Israel \\ and \\ School of Mathematics, Institute for Advanced Study, Princeton, NJ 08540, USA \\ (e-mail: nogaa@tau.ac.il)
}

Received 16 July 2016; revised 19 August 2017; first published online 9 October 2017

\begin{abstract}
We prove that there is a set $F$ in the plane so that the distance between any two points of $F$ is at least 1 , and for any positive $\varepsilon<1$, and every line segment in the plane of length at least $\varepsilon^{-1-o(1)}$, there is a point of $F$ within distance $\varepsilon$ of the segment. This is tight up to the $o(1)$-term in the exponent, improving earlier estimates of Peres, of Solomon and Weiss, and of Adiceam.
\end{abstract}

2010 Mathematics subject classification: Primary 51F99

Secondary 52C99

\section{Introduction}

A (planar) dense forest is a set $F \subset \mathbb{R}^{2}$ so that there exists a function $f:(0,1) \rightarrow \mathbb{R}^{+}$such that for any $\varepsilon \in(0,1)$ and any line segment $\ell$ in the plane of length at least $f(\varepsilon)$, there is a point $x \in F$ whose distance from $\ell$ is at most $\varepsilon$. A function $f$ satisfying the above is called a visibility function for $F$. The forest is uniformly discrete if there is a positive $r$ so that the distance between any two points of $F$ is at least $r$. The forest has finite density if there exists a finite $C$ such that for every $t \geqslant 1$ the number of points of $F$ in the ball of radius $t$ centred at the origin is at most $C t^{2}$. It is clear that any uniformly discrete forest has finite density, and that the converse is not true in general.

One reason for the terminology 'dense forest' is a visibility problem related to Pólya's Orchard Problem: see [5]. Indeed, a set $F$ is a dense forest as above if and only if, for any small $\varepsilon>0$, when we stand anywhere in a forest with tree trunks of radius $\varepsilon$ centred at the points of $F$, and look in any direction, we can never see further than distance $f(\varepsilon)$.

The problem of the existence of such forests, but only for a given fixed $\varepsilon>0$, was considered by Bishop in [4], where he described a construction, due to Peres, of a uniformly discrete dense

\footnotetext{
$\dagger$ Research supported in part by USA-Israeli BSF grant 2012/107, by ISF grant 620/13, by the Israeli I-Core programme and by the Fund for Mathematics.
} 
forest (for a fixed given $\varepsilon$ ) with visibility function $f(\varepsilon)=O\left(\varepsilon^{-4}\right)$. The same ideas can be used to show the existence of a dense forest of finite density with the above visibility function, but do not imply that there are uniformly discrete dense forests.

Solomon and Weiss [7] were the first to consider the problem of existence of a uniformly discrete dense forest, that is, a fixed uniformly discrete set $F$ that works for all values of $\varepsilon>0$. They proved the existence of such a forest using homogeneous dynamics and relying on a theorem of Ratner. Their proof provides no explicit bound for the visibility function. They also observed that the problem is closely related to a well-known question of Danzer, and asked if the $O\left(\varepsilon^{-4}\right)$ estimate (for the finite density case) can be improved. Adiceam [1] proved the existence of a dense forest with visibility function $c(\delta) \varepsilon^{-2-\delta}$, for any fixed $\delta>0$; however, his forest is of finite density but not uniformly discrete. Here we prove the existence of a uniformly discrete forest with visibility function $\varepsilon^{-1-o(1)}$. As $\Omega\left(\varepsilon^{-1}\right)$ is a trivial lower bound for the visibility function (even if we consider only vertical line segments), this is tight up to the $o(1)$-term in the exponent. A more precise statement of the result follows.

Theorem 1.1. There are absolute positive constants $r$ and $C$ so that there exists a uniformly discrete planar forest in which the distance between any two points is at least $r$, and the function

$$
f(\varepsilon)=\frac{2^{C} \sqrt{\log (1 / \varepsilon)}}{\varepsilon}
$$

is a visibility function.

The result is stronger than that in [7] as it gives an explicit nearly tight estimate for the visibility function, and is stronger than that in [1] in two respects: the forest is uniformly discrete and the visibility function is significantly smaller. On the other hand, the proof is probabilistic, whereas the forests of Solomon and Weiss in [7], of Peres in [4] and of Adiceam in [1] are constructed explicitly, and their properties are proved using techniques from dynamics (in [7]), Diophantine approximation (in [4]), and Fourier analysis (in [1]).

\section{Proof}

In this section we prove Theorem 1.1. Throughout the proof we make no attempt to optimize the absolute constants involved. The proof is probabilistic, combining some geometric considerations with the Lovász Local Lemma and a compactness argument. We will use the following basic version of the Local Lemma (see e.g. [3, Chapter 5]).

Lemma 2.1. Let $A_{i}, i \in I$ be a finite set of events in a probability space. Suppose that the probability of each event is at most $p$, and each event $A_{i}$ is mutually independent of all other events but at most $d$. If ep $(d+1) \leqslant 1$, then with positive probability none of the events holds.

Here is an outline of the proof of Theorem 1.1. We first define a family $H$ of horizontal strips in the plane, and a family $V$ of (broken) vertical strips. The horizontal strips are wider than the vertical ones, and the two families are defined in a way that ensures that any line segment that crosses a horizontal strip contains a significant portion in that strip, and this portion is not too 
close to its boundary, and is not too close to any vertical strip. Next we tile the plane by crosses, each consisting of 5 unit squares. Let $V C$ be the set of all crosses in the tiling that intersect a vertical strip, and let $H C$ denote the set of all crosses that intersect a horizontal strip and do not belong to $V C$. Replace each cross with a smaller cross consisting of all points of the cross whose distance from its boundary is at least, say, 0.05 . Our forest $F$ will contain exactly one point in each such smaller cross. Therefore it will be uniformly discrete (with $r=0.1$ ). Define a sequence $\varepsilon_{i}=2^{-i^{2}}$. Now number the horizontal and vertical strips by the non-zero integers, in order. Let $h_{p}$ be the horizontal strip number $p$ and let $v_{q}$ be the vertical strip number $q$. For each $i \geqslant 1$, the points in the (smaller) crosses corresponding to the horizontal and vertical strips $h_{p}, v_{q}$, where $p=2^{i} b, q=2^{i} b^{\prime}$, with $b, b^{\prime}$ odd, are used to handle all line segments of length $f\left(\varepsilon_{i}\right)$. This is done as follows.

In each smaller cross as above we place one point, randomly, uniformly and independently. Using compactness and the Local Lemma we show that there is such a placement that handles all line segments of length $f\left(\varepsilon_{i}\right)$. The union over $i$ of all these sets of points provides the required forest.

We proceed with the details. The absolute constants appearing throughout the proof can be easily improved.

For each (positive or negative) integer $p$, let $\ell_{p}$ denote the horizontal line $y=120 p$, and let $h_{p}$ denote the horizontal strip consisting of all points within distance at most 50 from $\ell_{p}$.

For each integer $q$ let $m_{q}$ denote the broken line obtained from the vertical line $x=120 q$ by adding to it detours inside the horizontal strips as follows. Each segment of the form $(x=$ $120 q, 120 p-20 \leqslant y \leqslant 120 p+20)$ is replaced by the following three segments:

- $120 q-80 \leqslant x \leqslant 120 q, y=120 p-20$,

- $x=120 q-80,120 p-20 \leqslant y \leqslant 120 p+20$,

- $120 q-80 \leqslant x \leqslant 120 q, y=120 p+20$.

Let $v_{q}$ be the vertical broken strip consisting of all points within distance 10 from $m_{q}$.

We say that a line segment $\ell$ crosses a horizontal strip $h_{p}$ if it contains points below and above it. Similarly, $\ell$ crosses a vertical broken strip $v_{q}$ if it contains points in its left and its right sides. Note that although each horizontal strip and each vertical strip intersect, any line segment that crosses a horizontal strip contains a sub-segment of length at least, say, 10 that lies in the horizontal strip and its distance from the boundary of the strip and from any vertical strip is at least 5. Similarly, any line segment that crosses a vertical strip contains a sub-segment of length at least 10 whose distance from the boundary of the strip is at least 5 .

A cross is a closed planar region consisting of a unit square whose vertices lie on the standard square lattice, and the 4 other unit lattice squares that share an edge with it. It is easy to see that there is a tiling of the plane with crosses; fix such a tiling. For any vertical broken strip $v_{q}$, with $q \in \mathbb{Z} \backslash\{0\}$, let $c v_{q}$ denote the set of all crosses in the tiling that intersect $v_{q}$. For any horizontal strip $h_{p}$, let $c h_{p}$ denote the set of all crosses that intersect $h_{p}$ and do not belong to any of the sets $c v_{q}, q \in \mathbb{Z} \backslash\{0\}$. Now replace each cross $x$ with a smaller subset of it, $x^{\prime}$, which is also crossshaped, consisting of all points of the cross $x$ of $\ell_{\infty}$-distance at least 0.05 from its boundary. For each $q \in \mathbb{Z} \backslash\{0\}$ put $c v_{q}^{\prime}=\left\{x^{\prime}: x \in c v_{q}\right\}$, and for each $p \in \mathbb{Z} \backslash\{0\}$, define $c h_{p}^{\prime}=\left\{x^{\prime}: x \in c h_{p}\right\}$. It is easy to see that any line segment $\ell$ that crosses a horizontal strip $h_{p}$ contains a subset of total length at least, say, 1 , where it crosses $h_{p}$, in which every point lies inside one of the smaller 
crosses in $c h_{p}^{\prime}$. Similarly, if $\ell$ crosses a vertical (broken) strip $v_{q}$, it must contain, where it crosses it, a subset of total length at least 1 consisting of points that lie inside one of the small crosses in $c v_{q}^{\prime}$.

Split the set of all small crosses in

$$
\bigcup_{p \neq 0} c h_{p}^{\prime} \cup \bigcup_{q \neq 0} c v_{q}^{\prime}
$$

into pairwise disjoint families $C_{1}, C_{2}, C_{3}, \ldots$ as follows. All crosses in $c h_{p}^{\prime}$ belong to $C_{i}$ if and only if the largest power of 2 that divides $p$ is $2^{i}$ (i.e. $|p|=2^{i} b$, where $b$ is odd). Similarly, the crosses in $c v_{q}^{\prime}$ belong to $C_{i}$ if and only if the largest power of 2 dividing $q$ is $2^{i}$.

Lemma 2.2. For each $i \geqslant 1$, there is a set of points $F_{i}$ consisting of exactly one point in each of the crosses in $C_{i}$, so that for any line segment $\ell$ of length at least $2^{i^{2}} 2^{i+15} i^{2}$ there is a point in $F_{i}$ whose distance from $\ell$ is at most $2^{-i^{2}}$.

Proof. Define $\varepsilon_{i}=2^{-i^{2}}$ and $\varepsilon=\varepsilon_{i} / 4$. Fix an $\varepsilon \times \varepsilon$ square grid in the plane, and note that by the triangle inequality it suffices to prove that there is a set $F_{i}$ consisting of one point in each cross in $C_{i}$, so that for any line segment $\ell$ of length at least $2^{i^{2}} 2^{i+15} i^{2}$ and at most, say, $2^{i^{2}} 2^{i+16} i^{2}$ whose end-points belong to the grid, there is a point of $F_{i}$ whose distance from $\ell$ is at most $\varepsilon$. The product space of all placements of one point in each (closed) cross is compact, by Tikhonov's theorem. For each fixed line segment $\ell$ as above, the set of placements that contain at least one point within distance at most $\varepsilon$ from $\ell$ is a closed set in this space. Our objective is to show that the intersection of all these sets is non-empty. By compactness it suffices to show that this is the case for the intersection of any finite family of these sets. Fix a finite family $\ell_{i}, i \in I$ of such line segments. Let $C^{\prime}$ be the set of all crosses in $C_{i}$ that intersect any of these lines. Place a random point, uniformly and independently, in each member of $C^{\prime}$. For each line segment $\ell$ in our family let $E_{\ell}$ be the event that none of these random points lies within distance at most $\varepsilon$ from $\ell$.

To complete the proof it suffices to show that with positive probability none of these events happens. This is done using the Local Lemma. We first bound the probability of each event $E_{\ell}$. Fix a line $\ell$ as above. Since its length is at least $2^{i^{2}} 2^{i+15} i^{2}$, it must cross at least

$$
\left\lfloor 2^{i^{2}} 2^{i+15} i^{2} / 2^{i+1}\right\rfloor>2^{i^{2}} 2^{13} i^{2}
$$

horizontal or vertical strips whose crosses belong to the family $C_{i}$. For each such crossing, it contains a subset of total length at least 1 inside the smaller crosses in $C^{\prime}$. Note that if a line segment contains a subset of length at least $w$ inside a small cross, then the probability that the random point selected in this cross lies within distance $\varepsilon$ from this segment is bigger than $w \varepsilon / 5$. The probability that this is not the case is thus smaller than $(1-\varepsilon w / 5)<e^{-\varepsilon w / 5}$. As the points selected in distinct small crosses are independent, the probability that none of the points in the crosses of $C^{\prime}$ intersected by $\ell$ lies within distance $\varepsilon$ from it is at most $e^{-\varepsilon \sum_{c} w_{c} / 5}$, where the sum in the exponent ranges over all crosses $c$ in $C^{\prime}$, and $w_{c}$ denotes the total length of the intersection of $\ell$ with $c$. As noted above, this sum is at least $2^{i^{2}} 2^{13} i^{2}$, and we thus conclude that

$$
\mathbb{P}\left[E_{\ell}\right] \leqslant e^{-\varepsilon 2^{i^{2}} 2^{13} i^{2} / 5}<e^{-2^{8} i^{2}},
$$

where we used the fact that $\varepsilon=\varepsilon_{i} / 4=2^{-i^{2}-2}$. 
Each event $E_{\ell}$ is mutually independent of all other events $E_{\ell^{\prime}}$ besides those corresponding to line segments $\ell^{\prime}$ in our family that intersect some of the smaller crosses in $C^{\prime}$ intersected by $\ell$. Any end-point of such a line $\ell^{\prime}$ lies within distance at most

$$
R=\frac{3}{2} 2^{i^{2}} 2^{i+16} i^{2}+4
$$

from the midpoint of $\ell$, implying that the lines $\ell^{\prime}$ whose number we have to bound have their end-points in a disc of area at most $\pi R^{2}$. The number of the $\varepsilon \times \varepsilon$ grid points inside this disc is roughly its area divided by $\varepsilon^{2}$, and thus the number of possibilities for choosing the two endpoints is smaller than

$$
10 \frac{81}{16} 2^{4 i^{2}} 2^{4 i+64} i^{8} / \varepsilon^{4}<2^{6} 2^{4 i^{2}} 2^{4 i+64} i^{8} 2^{4 i^{2}} 2^{8} \leqslant 2^{78} 2^{16 i^{2}} .
$$

Therefore each event $E_{\ell}$ is mutually independent of all other events but less than $D=2^{78} 2^{16 i^{2}}$, and as the probability $P$ of each such event satisfies $P<e^{-2^{8} i^{2}} \leqslant e^{-16 i^{2}} e^{-240}$ we conclude that the product $e P(D+1)$ is (much) smaller than 1, implying, by the Local Lemma (Lemma 2.1) that with positive probability none of the events $E_{\ell}$ in the family holds. By compactness, this completes the proof of the lemma.

To complete the proof of Theorem 1.1, let $F$ now be the union of all sets $F_{i}(i \geqslant 1)$, with $F_{i}$ as in Lemma 2.2. This is clearly a uniformly discrete forest, where the distance between any two points is at least 0.1 . For any $\varepsilon<1 / 2$, let $i$ be the minimum index so that $\varepsilon \geqslant \varepsilon_{i}=2^{-i^{2}}$. By this minimality, $\varepsilon<2^{-(i-1)^{2}}$. By the properties of $F_{i}$ there is a point within distance $\varepsilon_{i} \leqslant \varepsilon$ of any line segment of length

$$
2^{i^{2}} 2^{i+15} i^{2}=2^{(i-1)^{2}} 2^{3 i+14} i^{2}<\frac{1}{\varepsilon} 2^{3 \sqrt{\log (1 / \varepsilon)}+17}(\log (1 / \varepsilon)+1)^{2},
$$

implying that the function

$$
f(\varepsilon)=\frac{2^{O(\sqrt{\log (1 / \varepsilon)})}}{\varepsilon}
$$

is a visibility function for our forest. This completes the proof of the theorem.

\section{Concluding remarks and open problems}

(1) We have seen that there is a uniformly discrete forest with visibility function $f(\varepsilon)=\varepsilon^{-1-o(1)}$. Although the estimate $f(\varepsilon)=\varepsilon^{-1-o(1)}$ is tight up to the $o(1)$-term in the exponent, the most interesting open problem here is to decide whether or not the $o(1)$-term is required. As pointed out by Solomon and Weiss [7], the existence of a forest of finite density with visibility function $O(1 / \varepsilon)$ is equivalent to an old problem of Danzer about the existence of a set of finite density in the plane which intersects every convex set of area at least $\varepsilon$. The related question of whether there is such a uniformly discrete set was suggested by Boshernitzan. See [6] for some related results and problems.

(2) Like the results in [7] and in [1], our proof here can be extended to any dimension $d \geqslant 2$. To simplify the presentation we have described here only the planar case $d=2$. 
(3) The proof here applies the Lovász Local Lemma in its symmetric form. It is tempting to try to apply the non-symmetric lemma and prove simultaneously the desired result for all values of $\varepsilon_{i}$. This, however, does not work as the events corresponding to long segments depend on too many events corresponding to smaller segments. This is the reason for the extra complication arising from the need to handle each of the values $\varepsilon_{i}$ separately.

(4) As shown in [7], the existence of a dense forest of finite density with visibility function $O(1 / \varepsilon)$ (which is equivalent to a positive answer to Danzer's problem) is also equivalent to the statement that for any $\varepsilon>0$ there is a set $N_{\varepsilon}$ of $O(1 / \varepsilon)$ points in the unit square $[0,1]^{2}$ that intersects every rectangle of area $\varepsilon$ in the unit square. The area here is measured, of course, according to the usual Lebesgue measure. It is interesting to note that there are examples of probability distributions on $[0,1]^{2}$ for which there are no such sets $N_{\varepsilon}$. This follows from Theorem 1.3 in [2], which asserts that for any large $n$ there is a set $N$ of $n$ points in the unit square such that any set of points that intersects any line segment that contains at least $\alpha(n)$ of the points is of cardinality at least $n / 4$, where $\alpha(n)$ is a function growing (slowly) to infinity with $n$. Taking $\varepsilon=\alpha(n) / n$ and considering the discrete uniform probability distribution on the points of $N$ supplies an example of a distribution as above.

(5) After the completion of this short paper, and following a discussion with Gady Kozma, we found a variant of the proof of the main result which provides a better estimate for the $o(1)$ term in the exponent. A careful version of this alternative proof establishes the existence of a uniformly discrete planar forest with visibility function

$$
f(\varepsilon)=O\left(\frac{1}{\varepsilon} \log \left(\frac{1}{\varepsilon}\right) \log \log \left(\frac{1}{\varepsilon}\right)\right) .
$$

We believe that the original proof described here is instructive, but include also a brief outline of the alternative argument, without an optimized choice of the parameters, in a version that gives visibility function $O\left(\frac{1}{\varepsilon} \log ^{3}\left(\frac{1}{\varepsilon}\right)\right)$.

For every positive integer $i$, define $\varepsilon_{i}=100^{-100^{i}}$. The idea is to show that a small perturbation of a construction that works for all $\varepsilon \geqslant \varepsilon_{i}$ is, with positive probability, good for all $\varepsilon \geqslant \varepsilon_{i+1}$. The final construction is the limit of this sequence of constructions. Define

$$
\ell_{i}=100^{10+3 i} 100^{100^{i}} .
$$

We want to ensure that after step number $i$ the constructed set of points contains at least $100^{2 i}$ members within distance $\varepsilon_{i} / 4$ from any (discrete) line segment of length $\ell_{i}$ (and also works for all $\varepsilon \geqslant \varepsilon_{i}$ ). Note that the definition of a dense forest only requires one point within distance $\varepsilon_{i}$, but the stronger condition enables us to get a better estimate. The initial construction, for $i=1$, is obtained by following the basic approach described in Section 2, placing a random point in each small cross and applying the Local Lemma and compactness. Assuming we have a construction that satisfies the above for $\varepsilon_{i}$, shift each of its points, randomly and independently, by at most $\varepsilon_{i} / 2$, placing it in a uniformly chosen point within distance $\varepsilon_{i} / 2$ from its current location. It is not difficult to check that for any fixed line segment $L$ of length $\ell_{i+1}$, each of the points that lies within distance $\varepsilon_{i} / 4$ from $L$, before the shift, has probability exceeding $\varepsilon_{i+1} / 10 \varepsilon_{i}$ of lying within distance $\varepsilon_{i+1} / 4$ of $L$, after the random shift. Thus the number of shifted points satisfying that is 
a binomial random variable with expectation at least

$$
\frac{\ell_{i+1}}{\ell_{i}} 100^{2 i} \frac{\varepsilon_{i+1}}{10 \varepsilon_{i}} \geqslant \frac{100^{3} \cdot 100^{2 i}}{10}=10 \cdot 100^{2 i+2} .
$$

The probability that the value of this variable is less than $100^{2 i+2}$ can be bounded by the standard estimates for binomial distributions (see e.g. [3, Appendix A]), and this together with the Lovász Local Lemma and compactness provides the required construction for step number $i+1$. This completes the (brief sketch of the) proof.

\section{Acknowledgement}

I thank Yaar Solomon for helpful discussions, comments and references, and Gady Kozma for fruitful suggestions and discussions.

\section{References}

[1] Adiceam, F. (2016) How far can you see in a forest? IMRN 4867-4881.

[2] Alon, N. (2012) A non-linear lower bound for planar epsilon-nets. Discrete Comput. Geom. 47235 244.

[3] Alon, N. and Spencer, J. H. (2008) The Probabilistic Method, third edition, Wiley.

[4] Bishop, C. J. (2011) A set containing rectifiable arcs QC-locally but not QC-globally. Pure Appl. Math. Quart. 1 121-138.

[5] Pólya, G. (1976) Problems and Theorems in Analysis, Vol. 2, Springer.

[6] Solan, O., Solomon, Y. and Weiss, B. On problems of Danzer and Gowers and dynamics on the space of closed subsets of $\mathbb{R}^{d}$. IMRN to appear.

[7] Solomon, Y. and Weiss, B. (2014) Dense forests and Danzer sets. Ann. Sci. Éc. Norm. Supér 491053 1074. 